Trey: Ueber den Einfluss einiger Neutralsalze etc. 353

\title{
Ueber den Einfluss einiger Neutralsalze auf die Katalyse des Methylacetats durch Chlorwasserstoff- und Schwefelsäure;
}

von

\section{H. Trey.}

Die wesentlich veränderte Wirkung der Säuren durch die Gegenwart ihrer Neutralsalze ist bereits früher von W. Ostwald ${ }^{1}$ ) einer eingehenden Untersuchung unterzogen worden. Eine befriedigende Erklärung für den von den Salzen ausgeübten Einfluss wurde aber bisher noch nicht gefunden, daher unternahm ich auf Veranlassung von Herrn Prof. Dr. W. Ostwald vorliegende Arbeit, um durch einen weitern experimentellen Beitrag möglicherweise die Lösung dieses Problems zu fördern.

Zur Untersuchung gelangten die Salz- und Schwefelsäure mit den Salzen der Alkalien und alkalischen Erden unter Anwendung der Methylacetat-Methode, und waren die Versuchsflüssigkeiten, deren ich mich bediente, folgende: Normale Chlorwasserstoff- and Schwefelsäure (1 Grm.-Aeq. Säure im Liter) und normale Säure, zu der $1 / 4,1 / 2,1$ u. s. w. Aequivalent Neutralsalz hinzugefügt worden. Die Sulfate und wägbaren Chloride wurden gewogen; von den hygroskopischen Chloriden erst concentrirte wässrige Lösungen hergestellt und diese dann, nach Ermittelung ihres Chlorgehalts durch $1 / 10$ normale Silberlösung und Bestimmung des spec. Grew., mit Wasser und fünffach normaler Säure auf die normale Concentration gebracht. Ferner wurde ein Theil der normalen Lösungen aufs $2^{1} / 2$ fache, 5 fache und $12^{1} / 2$ fache verdünnt. In Folgendem wiederhole ich kurz das bereits früher mitgetheilte Verfahren.

1) Dies. Journ. [2] 23, 209. (188.1.) 
354 Trey: Ueb. d. Einfluss einiger Nentralsalze auf die

Je $10 \mathrm{Ccm}$. einer der obigen Flüssigkeiten wurden in ein Fläschchen pipettirt, dasselbe in den Thermostaten $\left(25^{\circ}\right)$ gebracht, und nachdem der Inhalt der Gläschen die gleiche Temperatur erlangt, $1 \mathrm{Ccm}$. Methylacetat hinzugefügt, das Fläschchen verschlossen, 1/2 Minute geschüttelt und wieder in den Thermostaten zurückgestellt. Nach Verlauf einer passenden Zeit wurde dann in Intervallen von 10 Minuten $1 \mathrm{Ccm}$. der Lösung mit einer feinen Pipette herausgenommen und mit verdünntem Barytwasser (etwa $1 / 20$ normal) titrirt, wobei die Zunahme $x$ der freien Säure ein Maass für den Zerfall des Methylacetats giebt. Nach Verlauf einer entsprechend längeren Zeit wird schliesslich der Endwerth b bestimmt.

In den folgenden Tabellen enthalten die Spalten derselben nach einander 1. die Zeit $t, 2,3$. und 4. die experimentell gefundenen Werthe $x$, sowie deren Mittel, reducirt auf ${ }^{1} / 20$ normalen Barytwassers, 5 . die Grösse $\log b-\log (b-x)$, 6. den vorigen Werth, dividirt durch $t-1$. Die Werthe von $x$ und $b$ sind mit 100 multiplicirt und die Berechnung mit Zuhülfenahme der von $W$. Ostwald veröffentlichten Tabelle $\left.{ }^{1}\right)$ über den Werth $\log b-\log (b-x)$ ausgeführt.

1. Chlorwasserstoffsäure.

Tab. I. HCl (1 Lit.)

\begin{tabular}{|c|c|c|c|c|c|}
\hline$t$ & I & II & Mittel & $\log b-\log (b-x)$ & Const. \\
\hline 120 & 1026 & 1027 & 1027 & 3674 & 30,87 \\
\hline 130 & 1086 & 1085 & 1086 & 4020 & 31,16 \\
\hline 140 & 1137 & 1139 & 1138 & 4349 & 31,29 \\
\hline 150 & 1185 & 1182 & 1184 & 4661 & 31,28 \\
\hline 160 & 1229 & 1224 & 1227 & 4976 & 31,30 \\
\hline 170 & 1272 & 1270 & 1271 & 5324 & 31,50 \\
\hline 180 & 1309 & 1306 & 1308 & 5640 & 31,51 \\
\hline$\infty$ & 1799 & 1801 & $\mid 1799$ & - & 31,27 \\
\hline
\end{tabular}

1) Dies. Journ. [2] 29, 406 (1884). 
Katalyse d. Methylacetats durch Chlorwasserstoffs.etc. 355

Tab. II. HCl (1 Lit.) $2 \frac{1}{2}$ fache Verdünnung.

\begin{tabular}{|c|c|c|c|}
\hline$t$ & I & III & Mittel \\
\hline 300 & 994 & 998 & 996 \\
\hline 310 & 1022 & 1021 & 1022 \\
\hline 320 & 1043 & 1042 & 1043 \\
\hline 330 & 1065 & 1062 & 1064 \\
\hline 340 & 1083 & 1084 & 1084 \\
\hline 350 & 1099 & 1103 & 1101 \\
\hline 360 & 1122 & 1124 & 1123 \\
\hline \multirow[t]{2}{*}{$\infty$} & 1807 & 1809 & $|1809|$ \\
\hline & 1810 & 1807 & \\
\hline
\end{tabular}

$\begin{array}{cc}\log b-\log (b-x) & \text { Coust. } \\ 3474 & 11,62 \\ 3615 & 11,70 \\ 3733 & 11,70 \\ 3853 & 11,71 \\ 3971 & 11,71 \\ 4073 & 11,67 \\ 4212 & 11,73 \\ - & 11,69\end{array}$

Tab. III. HCl (1 Lit.) 5 fache Verdünnung.

$\begin{array}{rrrrrr}600 & 982 & 994 & 988 & 3366 & 5,62 \\ 610 & 999 & 999 & 999 & 3423 & 5,62 \\ 620 & 1006 & 1011 & 1009 & 3476 & 5,62 \\ 630 & 1019 & 1022 & 1021 & 3539 & 5,63 \\ 640 & 1030 & 1031 & 1031 & 3593 & 5,62 \\ 650 & 1039 & 1039 & 1039 & 3636 & 5,60 \\ 660 & 1051 & 1051 & 1051 & 3693 & 5,60 \\ \infty & 1831 & 1832 & |1832| & - & 5,62\end{array}$

Tab. IV. HCl (1 Lit.) 121/2 fache Verdünnung.

$\begin{array}{rrrrcr}1460 & 946 & 943 & 945 & 3204 & 2,20 \\ 1470 & 957 & 956 & 957 & 3265 & 2,22 \\ 1480 & 966 & 964 & 965 & 3306 & 2,24 \\ 1490 & 966 & 966 & 966 & 3311 & 2,22 \\ 1500 & 966 & 972 & 969 & 3326 & 2,22 \\ 1510 & 971 & 976 & 974 & 3352 & 2,22 \\ 1520 & 976 & 979 & 978 & 3372 & 2,22 \\ \infty & 1809 & 1811 & \underline{\mid 1811} & - & -2,22\end{array}$

2. Ohlorwasserstoffsäure und Chlornatrium.

Tab. V. HCl (1 Lit.) $+1 / 4 \mathrm{NaCl}$.

$\begin{array}{rrrrrr}100 & 843 & 942 & 943 & 3236 & 32,69 \\ 110 & 1004 & 1008 & 1006 & 3570 & 32,75 \\ 120 & 1067 & 1066 & 1067 & 3919 & 32,93 \\ 130 & 1120 & 1113 & 1117 & 4229 & 32,78 \\ 140 & 1165 & 1160 & 1163 & 4534 & 32,62 \\ 150 & 1213 & 1212 & 1213 & 4892 & 32,83 \\ 160 & 1257 & 1249 & 1253 & 5201 & 32,71 \\ \infty & 1791 & 1793 & \overline{\mid 1795} & - & 32,76\end{array}$


356 Trey: Ueb. d. Einfluss einiger Neutralsalze auf die

Tab. VI. HCl $(1$ Lit. $)+1 / 4 \mathrm{NaCl} .21 / 2$ fache Verdünnung.

\begin{tabular}{rrrrcr}
$t$ & \multicolumn{1}{|c}{ I } & \multicolumn{1}{l|}{$\boldsymbol{x}$} & Mittel & $\log b-\log (b-x)$ & Const. \\
250 & 878 & 886 & 882 & 2899 & 11,64 \\
260 & 905 & 913 & 909 & 3027 & 11,69 \\
270 & 933 & 943 & 938 & 3169 & 11,78 \\
280 & 958 & 964 & 961 & 3284 & 11,77 \\
290 & 979 & 986 & 983 & 3399 & 11,76 \\
300 & 1004 & 1014 & 1009 & 3538 & 11,83 \\
310 & 1023 & 1034 & 1029 & 3647 & 11,80 \\
$\infty$ & 1798 & 1829 & $\underline{\underline{1811}}$ & - & 11,75
\end{tabular}

Tab. VII. $\mathrm{HCl}$ (1 Lit.) $+1 / 4 \mathrm{NaCl}$. 5 fache Verdünnung.

$\begin{array}{rrrrcc}540 & 908 & 916 & 912 & 3010 & 5,58 \\ 550 & 925 & 928 & 927 & 3082 & 5,61 \\ 560 & 935 & 939 & 937 & 3131 & 5,60 \\ 570 & 945 & 949 & 947 & 3181 & 5,59 \\ 580 & 959 & 963 & 961 & 3250 & 5,61 \\ 590 & 975 & 978 & 977 & 3331 & 5,66 \\ 600 & 985 & 986 & 986 & 3378 & 5,64 \\ \infty & 1817 & 1831 & \underline{\mid 1824} & - & 5,61\end{array}$

Tab. VIII. $\mathrm{HCl}(1 \mathrm{Lit}$ ) $+1 / 4 \mathrm{NaCl}$. 12/2 fache Verdünnung.

$\begin{array}{rrrrrr}1470 & 969 & 973 & 971 & 3255 & 2,22 \\ 1480 & 975 & 979 & 977 & 3285 & 2,22 \\ 1490 & 981 & 983 & 982 & 3311 & 2,22 \\ 1500 & 985 & 991 & 988 & 3341 & 2,23 \\ 1510 & 995 & 996 & 996 & 3382 & 2,24 \\ 1520 & 1000 & 1001 & 1001 & 3407 & 2,24 \\ 1530 & 1004 & 1006 & 1005 & 3428 & 2,24 \\ \infty & 1840 & 1840 & \mid 1 \overline{841} & - & 2,23\end{array}$

Tab. IX. HCl (1 Lit.) + $1 \mathrm{NaCl}$.

$\begin{array}{llllll}105 & 1023 & 1024 & 1024 & 3790 & 36,44 \\ 115 & 1092 & 1074 & 1083 & 4153 & 36,43 \\ 125 & 1147 & 1146 & 1147 & 4588 & 37,00 \\ 135 & 1197 & 1193 & 1195 & 4941 & 36,87 \\ 145 & 1245 & 1240 & 1243 & 5327 & 36,99 \\ 155 & 1286 & 1289 & 1288 & 5722 & 37,16 \\ 165 & 1333 & 1321 & 1327 & 6098 & 37,18 \\ \infty & 1768 & 1751 & \underline{\mid 1759 !} & - & 36,87\end{array}$


Katalyse d. Methylacetats durch Chlorwasserstoffs. etc. 357

Tab. X. HCl (1 Lit.) $+1 \mathrm{NaCl} .2 \frac{1}{2}$. fache Verdünnung.

\begin{tabular}{|c|c|c|c|c|c|}
\hline \multirow[b]{2}{*}{$t$} & \multicolumn{3}{|c|}{$x$} & \multirow[b]{2}{*}{$\log b-\log (b-x)$} & \multirow[b]{2}{*}{ Const } \\
\hline & $\widetilde{\mathrm{I}}$ & II & Mittel & & \\
\hline 240 & 876 & 877 & 877 & 2933 & 12,27 \\
\hline 250 & 901 & 901 & 901 & 3050 & 12,25 \\
\hline 260 & 928 & 930 & 929 & 3190 & 12,32 \\
\hline 270 & 950 & 949 & 950 & 3297 & 12,26 \\
\hline 280 & 973 & 972 & 973 & 3418 & 12,25 \\
\hline 290 & 996 & 997 & 997 & 3548 & 12,28 \\
\hline 300 & 1017 & 1018 & 1018 & 3665 & 12,26 \\
\hline$\infty$ & 1789 & 1783 & 1786 & - & 12,27 \\
\hline & 1789 & 1783 & & - & \\
\hline
\end{tabular}

Tab. XI. $\mathrm{HCl}(1 \mathrm{Lit})+.1 \mathrm{NaCl} .5$ fache Verdünnung.

$\begin{array}{rrrrrr}490 & 859 & 858 & 859 & 2802 & 5,73 \\ 500 & 873 & 873 & 873 & 2866 & 5,74 \\ 510 & 883 & 885 & 884 & 2918 & 5,73 \\ 520 & 900 & 901 & 901 & 2998 & 5,78 \\ 530 & 910 & 912 & 911 & 3047 & 5,76 \\ 540 & 923 & 923 & 923 & 3105 & 5,76 \\ 550 & 935 & 936 & 936 & \mathbf{3 1 7 0} & 5,77 \\ \infty & 1803 & 1809 & \underline{1807 \mid} & - & 5,75\end{array}$

Tab. XII. $\mathrm{HCl}$ (1 Lit.) $+1 \mathrm{NaCl} .12 \frac{1}{2}$ fache Verdünnung.

$\begin{array}{rrrrcr}1470 & 945 & 946 & 946 & 3217 & 2,19 \\ 1480 & 952 & 948 & 950 & 3237 & 2,20 \\ 1490 & 955 & 955 & 955 & 3263 & 2,19 \\ 1500 & 960 & 961 & 961 & 3293 & 2,20 \\ 1510 & 964 & 963 & 964 & 3309 & 2,19 \\ 1520 & 970 & 969 & 970 & 3340 & 2,20 \\ 1530 & 975 & 972 & 974 & 3360 & 2,20 \\ \infty & 1810 & 1808 & \underline{|1808|} & - & 2,20\end{array}$

Tab. XIII. HCI (1 Lit. $)+4 \mathrm{NaCl}$.

$\begin{array}{rrrrrr}40 & 634 & 626 & 630 & \mathbf{2 0 3 6} & 52,21 \\ \mathbf{5 0} & \mathbf{7 6 0} & \mathbf{7 4 6} & \mathbf{7 5 3} & \mathbf{2 5 7 6} & \mathbf{5 2 , 5 7} \\ 60 & 867 & 855 & 861 & 3112 & 52,75 \\ \mathbf{7 0} & 964 & 949 & 957 & \mathbf{3 6 5 1} & 52,91 \\ 80 & \mathbf{1 0 4 5} & \mathbf{1 0 4 4} & \mathbf{1 0 4 5} & \mathbf{4 2 1 3} & \mathbf{5 3 , 3 3} \\ 90 & 1118 & \mathbf{1 1 0 5} & \mathbf{1 1 1 2} & 4694 & \mathbf{5 2 , 7 4} \\ 100 & \mathbf{1 1 9 2} & \mathbf{1 1 8 2} & \mathbf{1 1 8 7} & \mathbf{5 3 0 7} & \mathbf{5 3 , 6 1} \\ \infty & \mathbf{1 6 8 7} & \mathbf{1 6 7 6} & \underline{\mid 1683} & - & -52,87\end{array}$


358 Trey: Ueb. d. Einfluss einigerNentralsalze auf die

Tab. XIV. $\mathrm{HCl}(1 \mathrm{Lit})+.4 \mathrm{NaCl} \cdot 21 / 2$ fache Verdünnung.

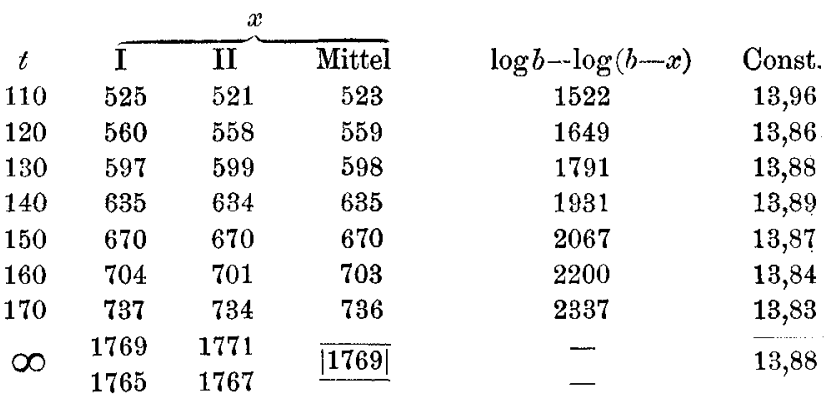

Tab. XV. HCl (1 Lit.) + $4 \mathrm{NaCl} .5$ fache Verdünnung.

$\begin{array}{rrrrrr}320 & 648 & 646 & 647 & 1937 & 6,07 \\ 330 & 662 & 663 & 663 & 1998 & 6,07 \\ 340 & 678 & 676 & 677 & 2052 & 6,05 \\ 350 & 696 & 695 & 696 & 2126 & 6,09 \\ 360 & 713 & 712 & 713 & 2194 & 6,11 \\ 370 & 724 & 727 & 726 & 2246 & 6,09 \\ 380 & 744 & 745 & \mathbf{7 4 5} & 2324 & 6,13 \\ \infty & 1799 & 1797 & \overline{|1798|} & - & 6,09\end{array}$

Tab. XVI. HCl $(1$ Lit. $)+4 \mathrm{NaCl} .12 \frac{1}{2}$ fache Verdünnung.

$\begin{array}{rrrrrr}1270 & 836 & 834 & 835 & 2718 & 2,14 \\ 1280 & 845 & 843 & 844 & 2759 & 2,16 \\ 1290 & 847 & 846 & 847 & 2773 & 2,15 \\ 1300 & 858 & 850 & 854 & 2805 & 2,16 \\ 1310 & 860 & 854 & 857 & 2818 & 2,15 \\ 1320 & 861 & 859 & 860 & 2833 & 2,15 \\ 1330 & 864 & 864 & 864 & 2851 & 2,15 \\ \infty & 1793 & 1796 & \underline{|1795|} & - & 2,15\end{array}$

3. Chlorwasserstoffsäure und Chlorkalium.

Tab. XVII. HCl (1 Lit.) $+1 / 4 \mathrm{KCl}$.

$\begin{array}{rrrrrr}100 & 934 & 931 & 933 & 3152 & 31,84 \\ 110 & 1005 & 991 & 998 & 3487 & 31,99 \\ 120 & 1056 & 1053 & 1055 & 3805 & 31,97 \\ 130 & 1111 & 1105 & 1108 & 4121 & 31,95 \\ 140 & 1162 & 1153 & 1158 & 4443 & 31,96 \\ 150 & 1205 & 1197 & 1201 & 4741 & 31,82 \\ 160 & 1247 & 1287 & 1242 & 5044 & 31,72 \\ \infty & 1816 & 1807 & {[1808]} & - & 31,89\end{array}$


Katalyse d. Methylacetats durch Chlorwasserstoffs. etc. 359

Tab. XVIII. $\mathrm{HCl}(1 \mathrm{Lit})+.1 / 4 \mathrm{KCl} .21 / 2$ fache Verdünnung.

\begin{tabular}{cccccc}
$t$ & \multicolumn{4}{c}{$x$} & \\
300 & 989 & $\mathbf{1 0 0 2}$ & 996 & $\log b-\log (b-x)$ & Const. \\
310 & 1021 & 1022 & 1022 & 3474 & 11,62 \\
320 & 1044 & 1048 & 1046 & 3615 & 11,70 \\
330 & 1065 & 1067 & 1066 & 3749 & 11,75 \\
340 & 1085 & 1087 & 1086 & 3865 & 11,75 \\
350 & 1106 & 1107 & 1107 & 3982 & 11,75 \\
360 & 1123 & 1123 & 1123 & 4111 & 11,78 \\
$\infty$ & 1809 & 1806 & 1809 & 4212 & 11,73 \\
& 1809 & 1810 & - & - & 11,73
\end{tabular}

Tab. XIX. HCl (1 Lit.) + 1/4 KCl. 5 fache Verdünnung.

$\begin{array}{rrrrrr}570 & 939 & 944 & 942 & 3189 & 5,61 \\ 580 & 955 & 959 & 957 & 3265 & 5,64 \\ 590 & 966 & 968 & 967 & 3316 & 5,63 \\ 600 & 977 & 976 & 977 & 3368 & 5,62 \\ 610 & 988 & 990 & 989 & 3430 & 5,63 \\ 620 & 998 & 1003 & 1001 & 3494 & 5,65 \\ 630 & 1010 & 1012 & 1011 & 3549 & 5,64 \\ \infty & 1807 & 1813 & \underline{\mid 1811]} & - & 5,63\end{array}$

Tab. XX. HCl (1 Lit.) + 1/4 KCl. 12/2 fache Verdünnung.

$\begin{array}{rrrrrr}1440 & 949 & 947 & 948 & 3185 & 2,21 \\ 1450 & 953 & 949 & 951 & 3201 & 2,21 \\ 1460 & 957 & 954 & 956 & 3225 & 2,21 \\ 1470 & 962 & 959 & 961 & 3250 & 2,21 \\ 1480 & 967 & 965 & 966 & 3275 & 2,21 \\ 1490 & 974 & 972 & 973 & 3300 & 2,22 \\ 1500 & 979 & 976 & 978 & 3337 & 2,23 \\ \infty & 1826 & 1819 & 1824 & - & 2,21\end{array}$

Tab. XXI. HCl (1 Lit.) $+1 \mathrm{KCl}$.

$\begin{array}{rrrrrr}80 & 828 & 823 & 826 & 2731 & 34,57 \\ 90 & 898 & 900 & 899 & 3079 & 34,60 \\ 100 & 965 & 965 & 965 & 3422 & 34,57 \\ 110 & 1028 & 1026 & 1027 & 3770 & 34,59 \\ 120 & 1090 & 1085 & 1088 & 4142 & 34,81 \\ 130 & 1141 & 1136 & 1139 & 4480 & 34,73 \\ 140 & 1191 & 1184 & 1188 & 4831 & 34,76 \\ \infty & 1770 & 1768 & |1770| & - & 34,66\end{array}$


360 Trey: Ueb. d. Einfluss einiger Neutralsalze auf die

Tab. XXII. HCl (1 Lit.) + $1 \mathrm{KCl}, 2 \frac{1}{2}$ fache. Verdünnung.

\begin{tabular}{rrrrcc}
$t$ & \multicolumn{1}{c}{ I } & \multicolumn{1}{c}{ II } & Mittel & $\log b-\log (b-x)$ & Const. \\
220 & 811 & 813 & 812 & $\mathbf{2 5 8 5}$ & 11,80 \\
230 & 841 & 841 & 841 & 2713 & 11,85 \\
240 & 869 & 870 & 870 & 2846 & 11,91 \\
250 & 895 & 894 & 895 & 2963 & 11,90 \\
260 & 919 & 919 & 919 & 3078 & 11,88 \\
270 & 946 & 945 & 946 & 3212 & 11,94 \\
280 & 966 & 974 & 970 & 3334 & 11,95 \\
$\infty$ & 1808 & 1810 & $\underline{|1810|}$ & - & 11,89
\end{tabular}

Tab. XXIII. HCl (1 Lit.) +1 KCl. 5 fache Verdünnung.

$\begin{array}{rrrrrr}470 & 809 & 816 & 813 & 2627 & 5,60 \\ 480 & 829 & 831 & 830 & 2703 & 5,64 \\ 490 & 837 & 843 & 840 & 2749 & 5,62 \\ 500 & 849 & 856 & 853 & 2809 & 5,63 \\ 510 & 862 & 869 & 866 & 2870 & 5,64 \\ 520 & 871 & 879 & 875 & 2912 & 5,61 \\ 530 & 887 & 892 & 890 & 2983 & 5,64 \\ \infty & 1788 & 1795 & \underline{|1791|} & - & 5,63\end{array}$

Tab. XXIV. HCl (1 Lit.) + $1 \mathrm{KCl}$. $12 \frac{1}{2}$ fache Verdünnung.

$\begin{array}{rrrrrr}1470 & 945 & 936 & 941 & 3146 & 2,14 \\ 1480 & 948 & 951 & 950 & 3191 & 2,16 \\ 1490 & 953 & 953 & 953 & 3205 & 2,15 \\ 1500 & 957 & 958 & 958 & 3230 & 2,16 \\ 1510 & 959 & 962 & 961 & 3245 & 2,15 \\ 1520 & 966 & 968 & 967 & 3266 & 2,15 \\ 1530 & 971 & 972 & 972 & 3301 & 2,16 \\ \infty & 1823 & 1829 & \overline{|1826|} & - & 2,15\end{array}$

Tab. XXV. HCl (1 Lit.) + 3 KCl.

$\begin{array}{rrrccl}40 & 530 & 533 & 532 & 1595 & 40,90 \\ 50 & 639 & 637 & 638 & 1997 & 40,76 \\ 60 & \mathbf{7 4 3} & \mathbf{7 3 4} & \mathbf{7 3 9} & 2417 & 40,97 \\ 80 & 908 & 901 & 905 & 3213 & 40,67 \\ 90 & 985 & 975 & 980 & 3627 & 40,75 \\ 100 & 1052 & 1045 & 1049 & 4045 & 40,86 \\ 110 & 1114 & 1107 & 1111 & 4459 & 40,91 \\ \infty & 1734 & 1727 & |1731| & - & -.- \\ & 1737 & 1725 & & - & 40,83\end{array}$


Katalyse d. Methylacetats durch Chlorwasserstoffs. etc. 361

Tab. XXVI. HCl (1 Lit.) + 3 KCl. $2 \frac{1}{2}$ fache Verdünnung.

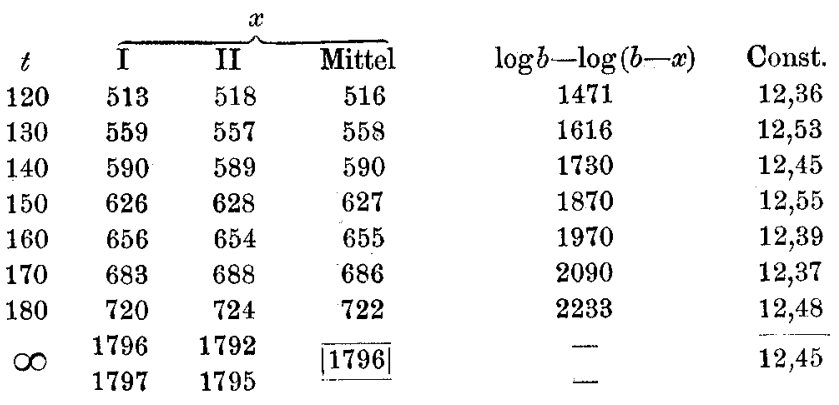

Tab. XXVII. HCl (1 Lit.) + 3KCl. 5 fache Verdünnung.

$\begin{array}{rrrrrr}310 & 597 & 601 & 599 & 1734 & 5,61 \\ 320 & 617 & 614 & 616 & 1795 & 5,63 \\ 330 & 627 & 628 & 628 & 1839 & 5,59 \\ 340 & 644 & 642 & 643 & 1893 & 5,58 \\ 350 & 660 & 655 & 658 & 1949 & 5,58 \\ 360 & 679 & 675 & 677 & 2020 & 5,63 \\ 370 & 689 & 690 & 690 & 2070 & 5,61 \\ \infty & 1820 & 1817 & |1820| & - & 5,60\end{array}$

Tab. XXVIII. HCl (1 Lit.) $+3 \mathrm{KCl}$. 12//2 fache Verdünnung.

$\begin{array}{rrrrrr}1200 & 794 & 791 & 793 & 2506 & 2,09 \\ 1210 & 802 & 797 & 799 & 2532 & 2,09 \\ 1220 & 807 & 802 & 805 & 2557 & 2,10 \\ 1230 & 812 & 807 & 810 & 2579 & 2,10 \\ 1240 & 819 & 812 & 816 & 2605 & 2,10 \\ 1250 & 823 & 816 & 820 & 2622 & 2,10 \\ 1260 & 828 & 822 & 825 & 2645 & 2,10 \\ \infty & 1816 & 1803 & \overline{[1809]} & - & 2,10\end{array}$

Da die hier erhaltenen Werthe nicht unmittelbar vergleichbar sind, weil durch das hinzugesetzte Salz das Volumen vermehrt worden, die Geschwindigkeit der Reaction mithin in demselben Verhältniss vermindert wird, so sind die für die Säure plus dem Salze erhaltenen Constanten, wie bereits a. a. 0 . ausgeführt' ${ }^{1}$, zu corrigiren. Um den

1) Dies. Journ. [2] 31, 227. (1885). 
362 Trey: Ueb. d. Einfluss einiger Neutralsalze auf die Ueberblick über die erhaltenen Zahlen zu erleichtern, stelle ich daher in folgender Tabelle die Mittelwerthe der Constanten, sowie deren corrigirte Werthe zusammen.

\begin{tabular}{|c|c|c|c|c|c|c|}
\hline$\underset{\text { (1 }}{\mathrm{HCl}}$ Lit.) & $\begin{array}{c}\mathrm{HCl} \\
(1 \mathrm{Lit}) \\
+1 / 4 \mathrm{NaCl}\end{array}$ & $\begin{array}{c}\text { HCl } \\
(1 \mathrm{Lit} .) \\
+1 \mathrm{NaCl} \\
\end{array}$ & $\begin{array}{c}\mathrm{HCl} \\
(1 \mathrm{Lit}) \\
+4 \mathrm{NaCl} \\
\end{array}$ & $\begin{array}{c}\mathrm{HCl} \\
(1 \mathrm{Lit} .) \\
+14 \mathrm{KCl} \\
\end{array}$ & $\begin{array}{c}\mathrm{HCl} \\
\text { (1 Lit.) } \\
+1 \mathrm{KCl} \\
\end{array}$ & $\begin{array}{c}\mathrm{HCl} \\
(1 \mathrm{Lit} .) \\
+3 \mathrm{KCl}\end{array}$ \\
\hline $\begin{array}{c}31,27 \\
-\end{array}$ & $\begin{array}{l}32,76 \\
32,87\end{array}$ & $\begin{array}{l}36,87 \\
37,49\end{array}$ & $\begin{array}{l}52,87 \\
57,62\end{array}$ & $\begin{array}{l}31,89 \\
32,16\end{array}$ & $\begin{array}{l}34,66 \\
35,05\end{array}$ & $\begin{array}{l}40,83 \\
44,60\end{array}$ \\
\hline \multicolumn{7}{|c|}{$2^{1} / 2$ fache Verdünnung. } \\
\hline $\begin{array}{c}11,69 \\
-\end{array}$ & $\begin{array}{l}11,75 \\
11,82\end{array}$ & $\begin{array}{l}12,27 \\
12,35\end{array}$ & $\begin{array}{l}13,88 \\
14,88\end{array}$ & $\begin{array}{l}11,73 \\
11,83\end{array}$ & $\begin{array}{l}11,89 \\
12,26\end{array}$ & $\begin{array}{l}12,45 \\
13,64\end{array}$ \\
\hline \multicolumn{7}{|c|}{5 fache Verdünnung. } \\
\hline 5,62 & $\begin{array}{l}5,61 \\
5,68\end{array}$ & $\begin{array}{l}5,75 \\
5,85\end{array}$ & $\begin{array}{l}6,09 \\
6,64\end{array}$ & $\begin{array}{l}5,63 \\
5,67\end{array}$ & $\begin{array}{l}5,63 \\
5,80\end{array}$ & $\begin{array}{l}5,60 \\
6,14\end{array}$ \\
\hline \multicolumn{7}{|c|}{$12^{1 / 2}$ fache Verdünnung } \\
\hline $\begin{array}{l}2,22 \\
-\end{array}$ & $\begin{array}{l}2,23 \\
2,24\end{array}$ & $\begin{array}{l}2,20 \\
2,24\end{array}$ & $\begin{array}{l}2,15 \\
2,34\end{array}$ & $\begin{array}{l}2,21 \\
2,22\end{array}$ & $\begin{array}{l}2,15 \\
2,22\end{array}$ & $\begin{array}{l}2,10 \\
2,30\end{array}$ \\
\hline
\end{tabular}

Berechnet man ferner das Verhältniss, in welchem die Reactionsgeschwindigkeit der Salzsäure durch das hinzugefügte Salz vermehrt wird, d. h., dividirt man die Constanten jeder Horizontalreihe durch die erste, der freien Säure entsprechende, Zahl, so erhält man folgende Tabelle:

$$
\begin{aligned}
& \begin{array}{l|l|l|l|l|l|l|}
1,000 \mid & 1,051 & 1,199 & 1,843 & 1,028 & 1,121 & 1,426
\end{array} \\
& 24 / 2 \text { fache Verdünnung. } \\
& \begin{array}{l|l|l|l|l|l|l|}
1,000 \mid & 1,011 & 1,056 & 1,273 & 1,012 & 1,049 & 1,167
\end{array} \\
& 5 \text { fache Verdünnung. } \\
& \begin{array}{l|l|l|l|l|l|l|}
1,000 \mid & 1,011 & 1,041 & 1,181 & 1,009 & 1,032 & 1,093
\end{array} \\
& 121 / 2 \text { fache Verdünnung. } \\
& \begin{array}{l|l|l|l|l|l|l|l}
1,000 & 1,009 & 1,009 & 1,054 & 1,000 & 1,000 & 1,005
\end{array}
\end{aligned}
$$

Wie man sieht, wird die Wirkung der Salzsäure durch hinzugefügtes Salz in den untersuchten Fällen auch nach dieser Methode proportional der Menge des Salzes verstärkt, ein Resultat, das sich in Uebereinstimmung mit den früheren Versuchen von W. Ostwald ${ }^{1}$ ) befindet. Mit weiter fortschreitender Verdünnung nimmt die verstärkende Wirkung

1) Dies. Journ. [2] 23, 219. (1880). 
Katalyse d. Methylacetats durch Chlorwasserstoffs. etc. 363

$a b$, aber in keinem einfachen Verhältniss, bleibt aber proportional der Menge des Salzes; bei 121/2 facher Verdünnung scheint auch noch ein Einfluss stattzufinden, doch sind die hier erhaltenen Werthe so klein, dass die Unterschiede bereits in das Bereich der möglichen Fehler fallen.

Die nun folgenden Versuche wurden angestellt, um den Einfluss verschiedener Salze kennen zu lernen, konnten aber leider nur in weit geringerem Umfang, als geplant, zur Ausführung gebracht werden, da das Titriren mit Phenolphtaleïn nur bei Zusatz von Salzen der Alkalien und alkalischen Erden sich als ausführbar erwies und es mir nicht gelang, einen auch bei Gegenwart anderer Salze brauchbaren Indicator aufzufinden.

Mit $\mathrm{LiCl}, \mathrm{MgCl}_{2}, \mathrm{CaCl}_{2}, \mathrm{SrCl}_{2}$ und $\mathrm{BaCl}_{2}$ habe ich nur je ein Verhältniss von Säure zu Salz und nur die fünffache Verdünnung untersucht. Die folgenden Tabellen enthalten die gefundenen Werthe.

4. Chlorwasserstoffäure und Chlorlithium.

Tab. XXIX. $\mathrm{HCl}(1$ Lit. $)+4 \mathrm{LiCl}$.

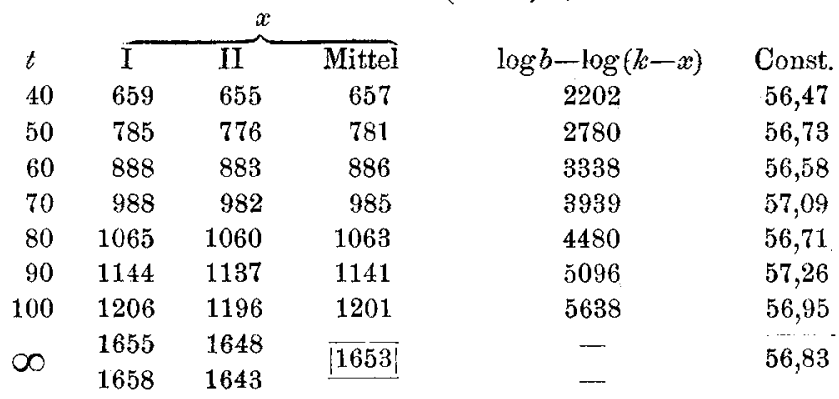

Tab. XXX. $\mathrm{HCl}(1 \mathrm{Lit})+.4 \mathrm{LiCl} .5$ fache Verdünnung.

$\begin{array}{rrrrrr}360 & 706 & 703 & 705 & 2151 & 5,99 \\ 370 & 716 & 716 & 716 & 2195 & 5,95 \\ 380 & 723 & 734 & 729 & 2247 & 5,93 \\ 390 & 748 & 748 & 748 & 2324 & 5,97 \\ 400 & 766 & 765 & 766 & 2398 & 6,00 \\ 410 & 778 & 777 & 778 & 2449 & 5,99 \\ 420 & 790 & 792 & 791 & 2504 & 5,98 \\ \infty & 1805 & 1801 & |1805| & - & 5,97\end{array}$


364 Trey: Ueb. den Einfluss einiger Nentralsalze auf die

5. Chlorwasserstoffsäure und Chlormagnesium.

Tab. XXXI. $\mathrm{HCl}(1$ Lit. $)+2 \mathrm{MgCl}_{2}$.

\begin{tabular}{rrrccl}
\multicolumn{1}{l}{$t$} & \multicolumn{1}{c}{$x$} & \multicolumn{1}{c}{$x$} & Mitttel & $\log b-\log (b-x)$ & Const. \\
40 & - & $\mathbf{7 4 1}$ & $\mathbf{7 4 1}$ & 2579 & 66,39 \\
50 & 865 & 861 & 863 & 3202 & 65,35 \\
60 & 976 & 976 & $\mathbf{9 7 6}$ & 3869 & 65,58 \\
70 & 1085 & 1078 & 1082 & 4607 & 66,77 \\
80 & $\mathbf{1 1 7 0}$ & 1165 & 1168 & $\mathbf{5 3 1 4}$ & 67,26 \\
90 & $\mathbf{1 2 4 0}$ & 1233 & $\mathbf{1 2 3 7}$ & 5976 & 67,15 \\
100 & $\mathbf{1 3 0 2}$ & 1296 & $\mathbf{1 2 9 9}$ & 6674 & 67,41 \\
$\infty$ & $\mathbf{1 6 5 7}$ & 1650 & $\underline{\mid \mathbf{1 6 5 5}}$ & - & 66,56
\end{tabular}

Tab. XXXII. $\mathrm{HCl}(1$ Lit. $)+2 \mathrm{MgCl}_{2}$. 5 fache Verdünnung.

$\begin{array}{rrrrrr}360 & 732 & 738 & 735 & 2268 & 6,32 \\ 370 & 751 & 753 & 752 & 2337 & 6,33 \\ 380 & 763 & 767 & 765 & 2391 & 6,31 \\ 390 & 779 & 785 & 782 & 2463 & 6,33 \\ 400 & 795 & 799 & 797 & 2527 & 6,33 \\ 410 & 810 & 813 & 812 & 2591 & 6,34 \\ 420 & 823 & 827 & 825 & 2649 & 6,32 \\ \propto & 1808 & 1807 & \underline{|1807|} & - & 6,33\end{array}$

6. Chlorwasserstoffsäure und Chlorcalcium.

\begin{tabular}{rrrrcr}
\multicolumn{5}{c}{ Tab. XXXIII. HCl (1 Lit.) +2 2CaCl. } \\
40 & 681 & 677 & 679 & 2293 & 58,80 \\
50 & 805 & 804 & 805 & 2894 & 59,06 \\
60 & 913 & 926 & 920 & 3525 & 59,75 \\
70 & 1009 & 1014 & 1012 & 4107 & 59,52 \\
80 & 1091 & 1098 & 1095 & 1698 & 59,47 \\
90 & 1162 & 1167 & 1165 & 5286 & 59,39 \\
100 & 1235 & 1233 & 1234 & 5945 & 60,05 \\
$\infty$ & 1661 & 1650 & $|1655|$ & - & 59,43
\end{tabular}

Tab. XXXIV. $\mathrm{HCl}(1 \mathrm{Lit})+.2 \mathrm{CaCl}_{2}$. 5 fache Verdünnung.

$\begin{array}{rrrrrr}\mathbf{3 6 0} & \mathbf{7 1 4} & \mathbf{7 2 0} & \mathbf{7 1 7} & \mathbf{2 2 3 7} & 6,23 \\ 370 & \mathbf{7 3 2} & \mathbf{7 3 2} & \mathbf{7 3 2} & \mathbf{2 2 9 9} & 6,23 \\ 380 & \mathbf{7 4 7} & \mathbf{7 4 7} & \mathbf{7 4 7} & \mathbf{2 3 6 1} & 6,23 \\ 390 & \mathbf{7 6 0} & \mathbf{7 6 1} & \mathbf{7 6 1} & \mathbf{2 4 2 0} & 6,22 \\ \mathbf{4 0 0} & \mathbf{7 7 9} & \mathbf{7 7 9} & \mathbf{7 7 9} & 2498 & 6,26 \\ \mathbf{4 1 0} & \mathbf{7 9 4} & \mathbf{7 9 2} & \mathbf{7 9 3} & \mathbf{2 5 5 9} & 6,26 \\ \mathbf{4 2 0} & 804 & 808 & 806 & \mathbf{2 6 1 7} & 6,25 \\ \infty & \mathbf{1 7 8 3} & 1777 & \overline{\mid \mathbf{1 7 8 1}} & - & 6,24 \\ & 1785 & 1777 & & - & \end{array}$


Katalyse d. Methylacetats dureh Chlorwasserstoffs. etc. 365

7. Ohlorwasserstoffsäure und Chlorstrontium.

Tab. XXXV. $\mathrm{HCl}(1$ Lit. $)+2 \mathrm{SrCl}_{2}$.

\begin{tabular}{rrrrcr}
\multicolumn{1}{c}{$t$} & \multicolumn{1}{c}{$x$} & Mittel & $\log b-\log (b-x)$ & Const. \\
40 & 681 & 683 & 682 & 2276 & 58,36 \\
50 & 805 & 803 & 804 & 2847 & 58,10 \\
60 & 920 & 911 & 916 & 3448 & 58,44 \\
70 & 1016 & 1013 & 1015 & 4057 & 58,80 \\
80 & 1107 & 1098 & 1103 & 4681 & 58,98 \\
90 & 1176 & 1168 & 1172 & 5242 & 58,90 \\
100 & 1231 & 1226 & 1229 & 5768 & 58,26 \\
$\infty$ & 1672 & 1666 & $\overline{|1672|}$ & - & 58,55
\end{tabular}

Tab. XXXVI. $\mathrm{HBl}(1$ Lit. $)+2 \mathrm{SrCl}_{2}$. 5 fache Verdünnung.

$\begin{array}{rrrrrr}375 & 739 & 738 & \mathbf{7 3 9} & 2313 & 6,19 \\ 385 & 758 & 753 & 756 & 2384 & 6,21 \\ 395 & \mathbf{7 7 1} & 766 & \mathbf{7 6 9} & \mathbf{2 4 3 8} & 6,19 \\ 405 & 785 & 783 & 784 & 2503 & 6,20 \\ 415 & 798 & 797 & 798 & 2563 & 6,19 \\ 425 & 816 & 811 & 814 & 2634 & 6,21 \\ 435 & 830 & 828 & 829 & 2701 & 6,22 \\ \infty & 1793 & 1787 & \overline{|1790|} & - & -6,20\end{array}$

8. Chlorwasserstoffsäure und Chlorbarium.

Tab. XXXVII. $\mathrm{HCl}\left(1\right.$ Lit.) $+1 \mathrm{BaCl}_{2}$.

$\begin{array}{rrrrrr}60 & 764 & 765 & 765 & 2498 & 42,34 \\ 70 & 856 & 849 & 853 & 2905 & 42,10 \\ 80 & 938 & 932 & 935 & 3321 & 42,04 \\ 90 & 1015 & 1007 & 1011 & 3737 & 41,99 \\ 100 & 1080 & 1077 & 1079 & 4167 & 42,09 \\ 110 & 1152 & 1171 & 1162 & 4742 & 43,50 \\ 120 & 1203 & 1223 & 1213 & 5136 & 43,16 \\ \propto & 1749 & 1745 & |1749| & - & 42,46\end{array}$

Tab. XXXVIII. $\mathrm{HCl}\left(1\right.$ Lit.) $+{ }_{1} \mathrm{BaCl}_{2}$. 5 fache Verdünnung.

$\begin{array}{rrrrrr}420 & 770 & 771 & 771 & 2438 & 5,82 \\ 430 & \mathbf{7 8 3} & 788 & 786 & 2502 & 5,83 \\ 440 & 796 & 802 & 799 & 2558 & 5,83 \\ 450 & 813 & 815 & 814 & 2624 & 5,84 \\ 460 & 823 & 828 & 826 & 2678 & 5,83 \\ 470 & 839 & 846 & 843 & 2754 & 5,87 \\ 480 & 852 & 857 & 855 & 2809 & 5,86 \\ \infty & 1792 & 1801 & -1795 & - & \overline{5,84}\end{array}$


366 Trey: Ueb. d. Einfluss einiger Neutralsalze cuf die

Zum Vergleich stelle ich auch hier wieder die entsprechenden Zahlen für Chlorwasserstoffsüure allein mit den corrigirten Werthen für Chlorwasserstoffsäure plus Salz zusammen, sowie die in obiger Weise berechneten Verhältnisszablen.

\begin{tabular}{|c|c|c|c|c|c|}
\hline $\mathrm{HCl}$ (1 Lit.) & $\begin{array}{c}\mathrm{HCl}(1 \mathrm{Lit}) \\
+4 \mathrm{LiCl}\end{array}$ & $\begin{array}{r}\mathrm{HCl}(1 \mathrm{Lit} \\
+2 \mathrm{MgCl}_{2} \\
\end{array}$ & $\begin{array}{l}\text { HCI (1 Lit.) } \\
+2 \mathrm{CaCl}\end{array}$ & $\begin{array}{c}\mathrm{HCl}(1 \mathrm{Lit} .) \\
+2 \mathrm{SrCl}_{2}\end{array}$ & $\left(\begin{array}{c}\mathrm{HCl} \text { (1 Lit.) } \\
+1 \mathrm{BaCl}_{2}\end{array}\right.$ \\
\hline 31,27 & $\begin{array}{l}56,83 \\
61,31\end{array}$ & $\begin{array}{l}66,56 \\
69,26\end{array}$ & $\begin{array}{l}59,43 \\
62,69\end{array}$ & $\begin{array}{l}58,55 \\
62,06\end{array}$ & $\begin{array}{l}42,46 \\
44,07\end{array}$ \\
\hline $\begin{array}{l}5,62 \\
-\end{array}$ & $\begin{array}{l}5,97 \\
6,44\end{array}$ & $\begin{array}{r}5 \text { fache } \\
6,33 \\
6,59\end{array}$ & $\begin{array}{l}\text { erdünnung. } \\
\begin{array}{c}6,24 \\
\mathbf{b}, \tilde{3} 6\end{array}\end{array}$ & $\begin{array}{l}6,20 \\
6,58\end{array}$ & $\begin{array}{l}5,84 \\
6,07\end{array}$ \\
\hline HCl (1 Lit.) & $\begin{array}{r}\mathrm{HCl}(1 \mathrm{Lit}) \\
+4 \mathrm{LiCl} \\
\end{array}$ & $\begin{array}{r}\mathrm{HCl}(1 \mathrm{Lit} \\
+2 \mathrm{MgCl}_{2} \\
\end{array}$ & $\begin{array}{r}\mathrm{HCl}(1 \mathrm{Lit}) \\
+2 \mathrm{CaCl}_{2}\end{array}$ & $\begin{array}{r}\mathrm{HCl}(1 \mathrm{Lit} .) \\
+2 \mathrm{SrCl}_{2} \\
\end{array}$ & $\begin{array}{r}\mathrm{HCl}(1 \mathrm{Lit} \\
+1 \mathrm{BrCl}\end{array}$ \\
\hline 1,000 & 1,961 & 2,215 & 2,005 & 1,985 & 1,409 \\
\hline \multicolumn{6}{|c|}{5 fache Verdünnung. } \\
\hline
\end{tabular}

Unter der Voraussetzung, dass auch diese Salze proportional ihrer Menge verstärkend wirken, stellt sich für alle hier untersuchten Chloride, hinsichtlich des beschleunigenden Einflusses, den dieselben auf die Katalyse des Methylacetats durch Chlorwasserstoffsäure ausüben, bezogen auf 1 Aeq. Chlorid, folgende Reihenfolge heraus:

\begin{tabular}{c|c|c|c|c|c|c}
\hline \hline $\mathrm{KCl}$ & $\mathrm{NaCl}$ & $\mathrm{BaCl}_{2}$ & $\mathrm{LiCl}$ & $\mathrm{SrCl}_{2}$ & $\mathrm{CaCl}_{2}$ & $\mathrm{MgCl}_{2}$ \\
\hline 1,121 & 1,199 & 1,205 & 1,240 & 1,246 & 1,251 & 1,304
\end{tabular}

Numerische Beziehungen lassen sich jedoch nicht feststellen, und will ich nur darauf hinweisen, dass, wenn man die untersuchten Chloride in die zugehörigen Gruppen einordnet, der beschleunigende Einfluss in umgekehrter Reihenfolge stattzuhaben scheint, als die Werthe der Atomgewichte in der Gruppe steigen.

\begin{tabular}{c|c|c|c|c|c|c}
\hline $\mathrm{IiCl}$ & $\mathrm{NaCl}$ & $\mathrm{KCl}$ & $\mathrm{MgCl}_{2}$ & $\mathrm{CaCl}_{2}$ & $\mathrm{SrCl}_{2}$ & $\mathrm{BaCl}_{2}$ \\
\hline 1,240 & 1,199 & 1,121 & 1,304 & 1,251 & 1,246 & 1,205
\end{tabular}


Katalyse d. Methylacetats durch Chlorwasserstoffs. etc. 367

Die von Reicher ${ }^{1}$ ) bei der Verseifung von Aethylacetat durch Basen erhaltenen Geschwindigkeitsconstanten ergeben dieselbe Reihenfolge, nämlich:

\begin{tabular}{c|c|c|c|c}
\hline \hline $\mathrm{Na}$ & $\mathrm{K}$ & $\mathbf{C a}$ & $\mathrm{Sr}$ & $\mathrm{Ba}$ \\
\hline 2,307 & 2,298 & 2,285 & 2,204 & 2,144
\end{tabular}

Ich lasse nun die mit Schwefelsäure und mit Schwefelsäure und deren Salzen angestellten Versuche folgen.

9. Schwefelsäure.

Tab. XXXIX. $\quad 1 / 2 \mathrm{H}_{2} \mathrm{SO}_{4}$ (1 Lit.)

\begin{tabular}{rrrrcc}
$t$ & \multicolumn{1}{c}{ I } & \multicolumn{1}{c}{ II } & Mittel & $\log b-\log (b-x)$ & Const. \\
180 & 896 & 896 & 896 & 2944 & 16,45 \\
190 & 932 & 929 & 931 & 3112 & 16,47 \\
200 & 969 & 974 & 972 & 3317 & 16,67 \\
210 & 999 & 997 & 998 & 3453 & 16,51 \\
220 & 1027 & 1026 & 1027 & 3608 & 16,48 \\
230 & 1060 & 1057 & 1059 & 3788 & 16,54 \\
240 & 1090 & 1089 & 1090 & 3968 & 16,60 \\
$\infty$ & 1817 & 1819 & $|1820|$ & - & 16,62
\end{tabular}

Tab. XL. ${ }_{1 / 2}^{1 / 2} \mathrm{H}_{2} \mathrm{SO}_{4}$ (1 Lit.) 21/2 fache Verdünnung.

$\begin{array}{rrrrrr}480 & 935 & 939 & 937 & 3147 & 6,57 \\ 490 & 958 & 956 & 957 & 3246 & 6,64 \\ 500 & 976 & 974 & 975 & 3338 & 6,69 \\ 510 & 989 & 990 & 990 & 3416 & 6,71 \\ 520 & 1000 & 997 & 999 & 3463 & 6,67 \\ 530 & 1014 & 1009 & 1012 & 3533 & 6,68 \\ 540 & 1023 & 1020 & 1022 & 3587 & 6,66 \\ \infty & 1823 & 1812 & \mid \overline{|1818|} & - & 6,66\end{array}$

1) Ann. Chem. 228, 257 (1885.) 
368 Trey: Ueb. d. Einfluss einiger Neutralsalze auf die Tab. XLI. $1 / 2 \mathrm{H}_{2} \mathrm{SO}_{4}(1$ Lit.) 5 fache Verdünnung.

\begin{tabular}{|c|c|c|c|c|c|}
\hline & & $x$ & & & \\
\hline$t$ & I & II & Mittel & $\log b-\log (b-x)$ & Const. \\
\hline 1090 & 1031 & 1035 & 1033 & 3647 & 3,35 \\
\hline 1100 & 1036 & 1037 & 1037 & 3669 & 3,34 \\
\hline 1110 & 1040 & 1044 & 1042 & 3698 & 3,34 \\
\hline 1120 & 1047 & 1048 & 1048 & 3732 & 3,34 \\
\hline 1130 & 1052 & 1057 & 1055 & 3771 & 3,34 \\
\hline 1140 & 1058 & 1062 & 1060 & 3800 & 3,34 \\
\hline 1150 & 1064 & 1068 & 1066 & 3834 & 3,34 \\
\hline$\infty$ & 1814 & 1819 & $|1818|$ & 一 & 3,34 \\
\hline & 1816 & 1820 & & & \\
\hline
\end{tabular}

Tab. XLII. $1 / 2 \mathrm{H}_{2} \mathrm{SO}_{4}$ (1 Lit.) 12/2 fache Verdünnung.

$\begin{array}{cccccc}2530 & 1012 & 1015 & 1014 & 3547 & 1,40 \\ 2540 & 1020 & 1019 & 1020 & 3579 & 1,41 \\ 2550 & 1025 & 1026 & 1026 & 3612 & 1,42 \\ 2560 & 1027 & 1027 & 1027 & 3617 & 1,41 \\ 2570 & 1030 & 1029 & 1030 & 3644 & 1,42 \\ 2580 & 1032 & 1032 & 1032 & 3645 & 1,41 \\ 2590 & 1034 & 1036 & 1035 & 3661 & 1,41 \\ \infty & 1817 & 1817 & {[1817} & - & 1,41\end{array}$

10. Schwefelsäure und schwefelsaures Natrium.

\begin{tabular}{llllcl}
\multicolumn{7}{c}{ Tab. XLIII. } & $1 / 2 \mathrm{H}_{2} \mathrm{SO}_{4}\left(1\right.$ Lit. $+1 / 8 \mathrm{Na}_{2} \mathrm{SO}_{4}$ \\
260 & 1049 & 1043 & 1046 & 3769 & 14,55 \\
270 & 1080 & 1078 & 1079 & 3962 & 14,73 \\
280 & 1109 & 1092 & 1101 & 4096 & 14,68 \\
290 & 1132 & 1121 & 1127 & 4261 & 14,74 \\
300 & 1155 & 1141 & 1148 & 4397 & 14,71 \\
310 & 1179 & 1168 & 1174 & 4573 & 14,80 \\
320 & 1201 & 1183 & 1192 & 4699 & 14,73 \\
$\infty$ & 1809 & 1794 & $\overline{\mid 1803]}$ & - & 14,71
\end{tabular}

Tab. XLIV. $1 / 2 \mathrm{H}_{2} \mathrm{SO}_{4}$ (1 Lit.) $+1 / 8 \mathrm{Na}_{2} \mathrm{SO}_{4} \cdot \quad 21 / 2$ fache Verdünnung.

$\begin{array}{cccccc}740 & 1141 & 1127 & 1134 & 4310 & 5,83 \\ 750 & - & 1139 & 1139 & 4343 & 5,80 \\ 760 & 1159 & 1147 & 1153 & 4436 & 5,85 \\ 770 & 1167 & 1153 & 1160 & 4482 & 5,83 \\ 780 & 1173 & 1167 & 1170 & 4551 & 5,84 \\ 790 & 1186 & 1172 & 1179 & 4613 & 5,85 \\ 800 & 1191 & 1179 & 1185 & 4655 & 5,83 \\ \infty & 1805 & 1801 & \underline{|1805|} & - & 5,83\end{array}$


Katalyse d. Methylacetats durch Chlorwasserstoffs. etc. 369

'Tab. XLV. ${ }_{1 / 2} \mathrm{H}_{2} \mathrm{SO}_{4}(1 \mathrm{Lit})+.1 / 8 \mathrm{Na}_{2} \mathrm{SO}_{4}$. 5 fache Verdünnung.

\begin{tabular}{|c|c|c|c|c|c|}
\hline \multirow[b]{2}{*}{$t$} & \multicolumn{3}{|c|}{$x$} & \multirow[b]{2}{*}{$\log b-\log (b-x)$} & \multirow[b]{2}{*}{ Const. } \\
\hline & I & II & Mittel & & \\
\hline 1630 & 1239 & 1234 & 1237 & 4918 & 3,02 \\
\hline 1640 & 1246 & 1242 & 1244 & 4970 & 3,03 \\
\hline 1650 & 1253 & 1246 & 1250 & 5016 & 3,04 \\
\hline 1660 & 1259 & 1250 & 1255 & 5054 & 3,05 \\
\hline 1670 & 1263 & 1255 & 1259 & 5085 & 3,05 \\
\hline 1680 & 1265 & 1257 & 1261 & 5100 & 3,04 \\
\hline 1690 & - & 1259 & 1259 & 5085 & 3,01 \\
\hline$\infty$ & $\begin{array}{l}1826 \\
1828\end{array}$ & 1823 & $|1825|$ & - & 3,04 \\
\hline
\end{tabular}

Tab. XLVI. $1 / 2 \mathrm{H}_{2} \mathrm{SO}_{4}(1$ Lit. $)+1 / 8 \mathrm{Na}_{2} \mathrm{SO}_{4} . \quad 12 / 2$ fache Verdünnung.

$\begin{array}{cccccc}3810 & 1238 & 1234 & 1236 & 4936 & 1,30 \\ 3820 & 1245 & 1236 & 1241 & 4975 & 1,30 \\ 3830 & 1246 & 1238 & 1242 & 4981 & 1,30 \\ 3840 & 1249 & 1239 & 1244 & 4996 & 1,30 \\ 3850 & 1254 & 1241 & 1248 & 5027 & 1,31 \\ 3860 & 1255 & 1243 & 1249 & 5035 & 1,31 \\ 3870 & 1255 & 1246 & 1251 & 5050 & 1,31 \\ & 1827 & 1813 & 1820 & - & 1,30\end{array}$

Tab. XLVII. $1 / 2 \mathrm{H}_{2} \mathrm{SO}_{4}(1 \mathrm{Lit})+.1 / 4 \mathrm{Na}_{2} \mathrm{SO}_{4}$.

$\begin{array}{rrrrrr}270 & 968 & 974 & 971 & 3359 & 12,49 \\ 280 & 1000 & 1001 & 1001 & 3518 & 12,61 \\ 290 & 1023 & 1023 & 1023 & 3639 & 12,59 \\ 300 & 1045 & 1043 & 1044 & 3757 & 12,57 \\ 310 & 1069 & 1066 & 1068 & 3896 & 12,61 \\ 320 & 1089 & 1090 & 1090 & 4029 & 12,63 \\ 330 & 1106 & 1107 & 1107 & 4134 & 12,57 \\ \infty & 1794 & 1809 & {[1803]} & - & 12,58\end{array}$

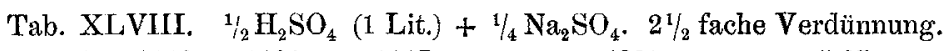

$\begin{array}{llllll}830 & 1113 & 1120 & 1117 & 4201 & 5,07 \\ 840 & 1122 & 1128 & 1125 & 4252 & 5,07 \\ 850 & 1129 & 1135 & 1132 & 4297 & 5,06 \\ 860 & 1137 & 1143 & 1140 & 4349 & 5,06 \\ 870 & 1144 & 1152 & 1148 & 4402 & 5,07 \\ 880 & 1154 & 1159 & 1157 & 4462 & 5,08 \\ 890 & 1161 & 1167 & 1164 & 4510 & 5,07 \\ \infty & 1800 & 1804 & {[\overline{1802}} & - & 5,07\end{array}$

Joumal f. prakt. Chemie [2] Bd. 34. 
370 Trey: Ueb. d. Einfluss einiger Neutralsalze auf die Tab. XLIX. ${ }^{1 / 2} \mathrm{H}_{2} \mathrm{SO}_{4}(1$ Lit. $)+1 / 4 \mathrm{Na}_{2} \mathrm{SO}_{4}$. 5 fache Verdünnung.

\begin{tabular}{|c|c|c|c|c|c|}
\hline \multirow{2}{*}{$t$} & \multicolumn{3}{|c|}{$x^{x}$} & \multirow[b]{2}{*}{$\log b-\log (b-x)$} & \multirow[b]{2}{*}{ Const. } \\
\hline & I & II & Mittel & & \\
\hline 1630 & 1145 & 1141 & 1143 & 4299 & 2,70 \\
\hline 1640 & 1164 & - & 1164 & 4436 & 2,71 \\
\hline 1650 & 1168 & - & 1168 & 4462 & 2,71 \\
\hline 1660 & 1170 & 1153 & 1162 & 4424 & 2,68 \\
\hline 1670 & 1172 & 1156 & 1164 & 4436 & 2,66 \\
\hline 1680 & 1178 & 1160 & 1169 & 4469 & 2,66 \\
\hline 1690 & 1181 & 1166 & 1175 & 4510 & 2,67 \\
\hline$\infty$ & 1825 & $\begin{array}{l}1812 \\
1813\end{array}$ & 1819 & - & 2,68 \\
\hline & 1825 & 1813 & & - & \\
\hline
\end{tabular}

Tab. L. ${ }_{1 / 2} \mathrm{H}_{2} \mathrm{SO}_{4}(1 \mathrm{Lit})+.1 / 4 \mathrm{Na}_{2} \mathrm{SO}_{4} .121 / 2$ fache Verdünnung.

$\begin{array}{cccccc}3830 & 1166 & 1170 & 1168 & 4498 & 1,18 \\ 3840 & 1177 & 1176 & 1177 & 4558 & 1,19 \\ 3850 & 1179 & 1177 & 1178 & 4565 & 1,19 \\ 3860 & 1179 & 1179 & 1179 & 4572 & 1,19 \\ 3870 & 1181 & 1183 & 1182 & 4593 & 1,19 \\ 3880 & 1182 & 1183 & 1183 & 4599 & 1,19 \\ 3890 & 1184 & 1184 & 1184 & 4607 & 1,19 \\ \infty & 1809 & 1813 & |1811| & - & 1,19\end{array}$

Tab. LI. ${ }_{1 / 2} \mathrm{H}_{2} \mathrm{SO}_{4}$ (1 Lit.) $+1 / 2 \mathrm{Na}_{2} \mathrm{SO}_{4}$.

$\begin{array}{rrrrrr}280 & 815 & 828 & 822 & 2619 & 9,41 \\ 290 & 842 & 866 & 854 & 2761 & 9,55 \\ 300 & 868 & 875 & 872 & 2843 & 9,51 \\ 310 & 887 & 893 & 890 & 2927 & 9,47 \\ 320 & 903 & 917 & 910 & 3023 & 9,48 \\ 330 & 931 & 936 & 934 & 3139 & 9,54 \\ 340 & 943 & 959 & 951 & 3224 & 9,51 \\ \infty & - & 1816 & \overline{\mid 1815]} & - & 9,50\end{array}$

Tab. LII. $1 / 2 \mathrm{H}_{2} \mathrm{SO}_{4}\left(1\right.$ Lit.) $+1 / 2 \mathrm{Na}_{2} \mathrm{SO}_{4} . \quad 21 / 2$ fache Verdünnung.

$\begin{array}{rrrrrr}780 & 921 & 934 & 928 & 3135 & 4,02 \\ 790 & 938 & 934 & 936 & 3175 & 4,02 \\ 800 & 941 & 942 & 942 & 3205 & 4,01 \\ 810 & 948 & 948 & 948 & 3235 & 4,00 \\ 820 & 952 & 956 & 954 & 3266 & 3,99 \\ 830 & 964 & 971 & 968 & 3338 & 4,03 \\ 840 & 973 & 972 & 973 & 3364 & 4,01 \\ \infty & 1799 & 1810 & \overline{1805 \mid} & - & 4,01\end{array}$


Katalyse d. Methylacetats durch Chlorwasserstoffs. etc. 371

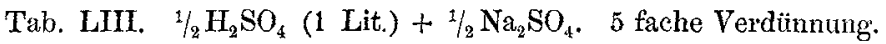

\begin{tabular}{cccccr}
$t$ & \multicolumn{1}{c}{$\mathrm{I}^{2}$} & II & Mittel & $\log b-\log (b-x)$ & Const. \\
1620 & 1002 & 999 & 1001 & 3477 & 2,15 \\
1630 & 1013 & 1006 & 1010 & 3525 & 2,16 \\
1640 & 1015 & 1008 & 1012 & 3436 & 2,16 \\
1650 & 1019 & 1018 & 1019 & 3573 & 2,17 \\
1660 & 1024 & 1021 & 1023 & 3595 & 2,17 \\
1670 & 1027 & 1025 & 1026 & 3612 & 2,16 \\
1680 & 1032 & 1028 & 1030 & 3634 & 2,16 \\
$\infty$ & 1822 & 1812 & $\underline{1817]}$ & - & $-2,16$
\end{tabular}

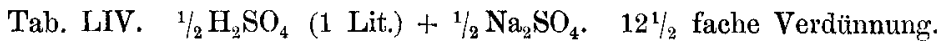

\begin{tabular}{|c|c|c|c|c|c|}
\hline 3780 & 1050 & 1051 & 1051 & 3758 & 1,00 \\
\hline 3790 & 1053 & 1055 & 1054 & 3775 & 1,00 \\
\hline 3800 & 1056 & 1058 & 1057 & 3793 & 1,00 \\
\hline 3810 & 1061 & 1061 & 1061 & 3816 & 1,00 \\
\hline 3820 & 1064 & 1062 & 1063 & 3827 & 1,00 \\
\hline 3830 & 1066 & 1065 & 1066 & 3843 & 1,00 \\
\hline 3840 & 1068 & 1069 & 1069 & 3862 & 1,01 \\
\hline$\infty$ & $\begin{array}{l}1816 \\
1813\end{array}$ & $\begin{array}{l}1815 \\
1815\end{array}$ & 1815 & - & 1,00 \\
\hline
\end{tabular}

Tab. LV. $1 / 2 \mathrm{H}_{2} \mathrm{SO}_{4}(1$ Lit. $)+1 \mathrm{Na}_{2} \mathrm{SO}_{4}$.

$\begin{array}{rrrrrr}270 & 621 & 615 & 618 & 1848 & 6,87 \\ 280 & 638 & 637 & 638 & 1924 & 6,90 \\ 290 & 653 & 653 & 653 & 1980 & 6,85 \\ 300 & 674 & 673 & 674 & 2062 & 6,90 \\ 310 & 690 & 686 & 688 & 2117 & 6,85 \\ 320 & 710 & 707 & 709 & 2201 & 6,90 \\ 330 & 730 & 727 & 729 & 2283 & 6,94 \\ \infty & 1781 & 1784 & |1783| & - & 6,89\end{array}$

Tab. LVI. $1 / 2 \mathrm{H}_{2} \mathrm{SO}_{4}(1 \mathrm{Lit})+.1 \mathrm{Na}_{2} \mathrm{SO}_{4} \cdot 21 / 2$ fache Verdünnung.

$\begin{array}{rrrrrr}800 & 751 & 750 & \mathbf{7 5 1} & 2376 & \mathbf{2 , 9 7} \\ 810 & 759 & \mathbf{7 5 6} & \mathbf{7 5 8} & 2408 & \mathbf{2 , 9 8} \\ 820 & 768 & \mathbf{7 5 8} & \mathbf{7 6 3} & \mathbf{2 4 2 9} & \mathbf{2 , 9 7} \\ 830 & \mathbf{7 7 4} & \mathbf{7 7 4} & \mathbf{7 7 4} & \mathbf{2 4 7 7} & \mathbf{2 , 9 9} \\ 890 & \mathbf{8 1 7} & \mathbf{8 1 5} & 816 & \mathbf{2 6 6 2} & 2,99 \\ 1040 & 912 & 906 & 909 & \mathbf{3 1 0 2} & 2,99 \\ 1050 & 924 & 914 & 919 & 3152 & \mathbf{3 , 0 1} \\ \infty & 1785 & 1773 & \underline{1781]} & - & -2,99\end{array}$


372 Trey: Ueb. d. Einfluss einiger Nentralsalze auf die Tab. LVII. $1 / 2 \mathrm{H}_{2} \mathrm{SO}_{4}(1$ Lit. $)+1 \mathrm{Na}_{2} \mathrm{SO}_{4} .5$ fache Verdünnung.

\begin{tabular}{cccccc}
$t$ & \multicolumn{1}{c}{ I } & II & Mittel & $\log b-\log (b-x)$ & Const. \\
1620 & 824 & 815 & 820 & 2618 & 1,62 \\
1630 & 832 & 817 & 825 & 2641 & 1,62 \\
1640 & 837 & 819 & 828 & 2654 & 1,62 \\
1650 & 842 & 827 & 835 & 2685 & 1,63 \\
1660 & 844 & 830 & 837 & 2694 & 1,62 \\
1670 & 846 & 835 & 841 & 2711 & 1,62 \\
1680 & 850 & 837 & 844 & 2725 & 1,62 \\
$\infty$ & 1811 & - & $-|1811|$ & - & 1,62
\end{tabular}

Tab. LVIII. $\quad 1 / 2 \mathrm{H}_{2} \mathrm{SO}_{4}$ (1 Lit.) $+1 \mathrm{Na}_{\sharp} \mathrm{SO}_{4}, \quad 12 \frac{1}{2}$ fache Verdünnung.

$\begin{array}{rrrrrr}3820 & 901 & 902 & 902 & 3010 & 0,79 \\ 3830 & 904 & 906 & 905 & 3025 & 0,79 \\ 3840 & 906 & 908 & 907 & 3034 & 0,79 \\ 3850 & 909 & 910 & 910 & 3049 & 0,79 \\ 3860 & 913 & 913 & 913 & 3064 & 0,79 \\ 3870 & 915 & 914 & 915 & 3074 & 0,79 \\ 3880 & 918 & 915 & 917 & 3083 & 0,79 \\ \infty & 1800 & 1807 & \mid \mathbf{1 8 0 4} & - & 0,79\end{array}$

Wie ersichtlich, wirken die Salze bei der Schwefelsäure in entgegengesetztem Sinne, als bei der Salzsäure. Während bei dieser durch die Gegenwart ihrer Neutralsalze eine beschleunigende Wirkung auf die Reactionsgeschwindigkeit der Säure resultirt, wird durch Hinzufügung der Sulfate die Reactionsgeschwindigkeit jener bedeutend vermindert. Stellt man auch hier die Werthe für die Säure allein mit den, wie oben corrigirten, Werthen für Säure plus Salz, sowie die berechneten Verhältnisszahlen zusammen, so ergiebt sich folgende Tabelle.

\begin{tabular}{|c|c|c|c|c|}
\hline $\begin{array}{l}1 / 2 \mathrm{H}_{2} \mathrm{SO}_{4} \\
\text { (1 Lit.) }\end{array}$ & $\begin{array}{c}1 / 2 \mathrm{H}_{2} \mathrm{SO}_{4} \text { (1Lit.) } \\
+1 / 8 \mathrm{Na}_{2} \mathrm{SO}_{4} \\
\end{array}$ & $\begin{array}{c}\left.1 / 2 \mathrm{H}_{2} \mathrm{SO}_{4} \text { (1Lit. }\right) \\
+\quad 1 / 4 \mathrm{Na}_{2} \mathrm{SO}_{4} \\
\end{array}$ & $\begin{array}{l}1 / 2 \mathrm{H}_{2} \mathrm{SO}_{4}(1 \mathrm{Lit} .) \\
+1 / 2 \mathrm{Na}_{3} \mathrm{SO}_{4} \\
\end{array}$ & $\begin{array}{c}1 / 2 \mathrm{H}_{2} \mathrm{SO}_{4}(1 \mathrm{Lit}) \\
+1 \mathrm{Na}_{2} \mathrm{SO}_{4}\end{array}$ \\
\hline 16,62 & 14,71 & 12,58 & 9,50 & 6,89 \\
\hline- & 14,71 & 12,64 & 9,62 & 7,08 \\
\hline \multicolumn{5}{|c|}{$21 / 2$ fache Verdünnung. } \\
\hline 6,66 & 5,83 & 5,07 & 4,01 & 2,99 \\
\hline - & 5,83 & 5,10 & 4,06 & 3,08 \\
\hline \multicolumn{5}{|c|}{5 fache Verdünnung. } \\
\hline 3,34 & 3,04 & 2,68 & 2,16 & 1,62 \\
\hline - & 3,04 & 2,69 & 2,19 & 1,67 \\
\hline
\end{tabular}




\begin{tabular}{|c|c|c|c|c|}
\hline $\begin{array}{c}1 / 2 \mathrm{H}_{2} \mathrm{SO}_{4} \\
(1 \mathrm{Iit})\end{array}$ & $\begin{array}{r}1 / 2 \mathrm{H}_{2} \mathrm{SO}_{4}(1 \mathrm{Lit} \\
+1 / 8 \mathrm{Na}_{2} \mathrm{SO} \\
\end{array}$ & t.) $\begin{array}{c}1 / 2 \mathrm{H}_{2} \mathrm{SO}_{4}(1 \mathrm{Lit} .) \\
+1 / 4 \mathrm{Na}_{2} \mathrm{SO}_{4}\end{array}$ & $\begin{array}{c}1{ }_{2} \mathrm{H}_{2} \mathrm{SO}_{4}(1 \mathrm{Lit} .) \\
+1 / 2 \mathrm{Na}_{2} \mathrm{SO}_{4} \\
\end{array}$ & $\begin{array}{c}1 / 2 \mathrm{H}_{2} \mathrm{SO}_{4}(1 \mathrm{Lit} .) \\
+1 \mathrm{Na}_{2} \mathrm{SO}_{4}\end{array}$ \\
\hline \multicolumn{5}{|c|}{$121 / 2$ fache Verdünnung. } \\
\hline 1,41 & 1,30 & 1,19 & 1,00 & 0,79 \\
\hline- & 1,30 & 1,20 & 1,01 & 0,81 \\
\hline 1,000 & 0,885 & 0,760 & 0,578 & 0,426 \\
\hline \multicolumn{5}{|c|}{$21 / 2$ fache Verdünnung. } \\
\hline 1,000 & 0,875 & $\quad 0,766$ & 0,610 & 0,463 \\
\hline \multicolumn{5}{|c|}{5 fache Verdünnung. } \\
\hline 1,000 & 0,910 & 0,805 & 0,656 & 0,500 \\
\hline \multicolumn{5}{|c|}{$12^{1} / 2$ fache Verdünnung. } \\
\hline 1,000 & 0,922 & 0,851 & 0,716 & 0,574 \\
\hline
\end{tabular}

Mit wachsender Salzmenge wird in allen untersuchten Fällen die Reactionsgeschwindigkeit der Schwefelsäure vermindert und zwar von $1 / 4$ bis zu 1 Aeq. Salz annähernd proportional, bei 2 Aeq. in geringerem Maasse. Mit zunehmender Verdünnung nimmt auch der verzögernde Einfluss ab, jedoch in keinem einfachen Verhältniss. Aus bereits angeführten Gründen musste ich auch bei der Schwefelsäure hinsichtlich des Einflusses anderer Salze mich auf die Untersuchung mit $\mathrm{K}_{2} \mathrm{SO}_{4}, \mathrm{Li}_{3} \mathrm{SO}_{4}$ und $\mathrm{MgSO}_{4}$ beschränken. Auch hier habe ich nur ein Verhältniss von Säure zu Salz und nur die fünffache Verdünnung untersucht. In den folgenden Tabellen gebe ich die erhaltenen Resultate.

11. Schwefelsäure und schwefelsaures Kalium.

\begin{tabular}{|c|c|c|c|c|c|}
\hline$t$ & $\widetilde{\mathrm{I}}$ & II & Mittel & $\log b-\log (b-x)$ & Const. \\
\hline 270 & 740 & 738 & 739 & 2278 & 8,47 \\
\hline 280 & 762 & 762 & 762 & 2372 & 8,50 \\
\hline 290 & 786 & 786 & 786 & 2472 & 8,55 \\
\hline 300 & 805 & 804 & 805 & 2553 & 8,54 \\
\hline 210 & 824 & 822 & 823 & 2631 & 8,52 \\
\hline 320 & 847 & 846 & 847 & 2739 & 8,59 \\
\hline 330 & 864 & 861 & 863 & 2811 & 8,54 \\
\hline$\infty$ & $\begin{array}{l}1811 \\
1810\end{array}$ & $\begin{array}{l}1811 \\
1811\end{array}$ & $\overline{1811}$ & - & 8,53 \\
\hline & & & & & r. 8,81 \\
\hline
\end{tabular}


374 Trey: Ueb. d. Einfluss einiger Neutralsalze auf die Tab. LX. $\quad 1 / 2 \mathrm{H}_{2} \mathrm{SO}_{4}(1$ Lit. $)+1 / 2 \mathrm{~K}_{2} \mathrm{SO}_{4} . \quad 5$ fache Verdünnung.

\begin{tabular}{rrrccr}
$t$ & \multicolumn{1}{|c}{ I } & \multicolumn{1}{|l}{} & Mittel & $\log b-\log (b-x)$ & Const. \\
1620 & 987 & 983 & 985 & 3390 & 2,09 \\
1630 & 994 & 992 & 993 & 3423 & 2,10 \\
1640 & 1000 & 997 & 999 & 3454 & 2,11 \\
1650 & 1001 & 1001 & 1001 & 3465 & 2,10 \\
1660 & 1006 & 1002 & 1004 & 3481 & 2,10 \\
1670 & 1009 & 1008 & 1009 & 3508 & 2,10 \\
1680 & 1014 & 1012 & 1013 & 3529 & 2,10 \\
$\infty$ & 1812 & 1829 & $\overline{|1821|}$ & - & $-2,10$ \\
& 1811 & 1829 & - & - & corr. 2,17
\end{tabular}

12. Schwefelsäure und schwefelsaures Lithium.

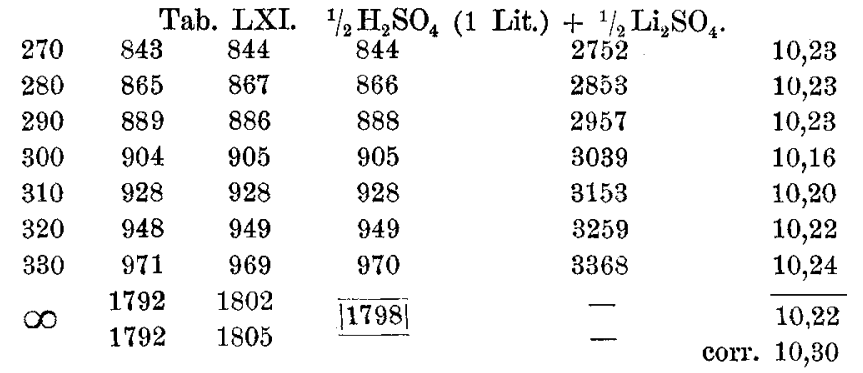

Tab. LXII. ${ }_{1 / 2} \mathrm{H}_{2} \mathrm{SO}_{4}$ (1 Lit.) $+{ }^{1 / 2} \mathrm{Li}_{2} \mathrm{SO}_{4} .5$ fache Verdünnung.

$\begin{array}{rrrrrr}1620 & 1008 & 1005 & 1007 & 3539 & 2,19 \\ 1630 & 1013 & 1007 & 1010 & 3555 & 2,19 \\ 1640 & 1019 & 1013 & 1016 & 3588 & 2,19 \\ 1650 & 1023 & 1015 & 1019 & 3604 & 2,19 \\ 1660 & 1027 & 1019 & 1023 & 3626 & 2,19 \\ 1670 & 1030 & 1023 & 1027 & 3648 & 2,19 \\ 1680 & 1033 & 1027 & 1030 & 3665 & 2,18 \\ \infty & 1812 & 1802 & \underline{|1807|} & - & 2,19 \\ & 1812 & 1802 & \underline{ } & - & \text { corr. } 2,21\end{array}$

13. Schwefelsäure und schwefelsaures Magnesium.

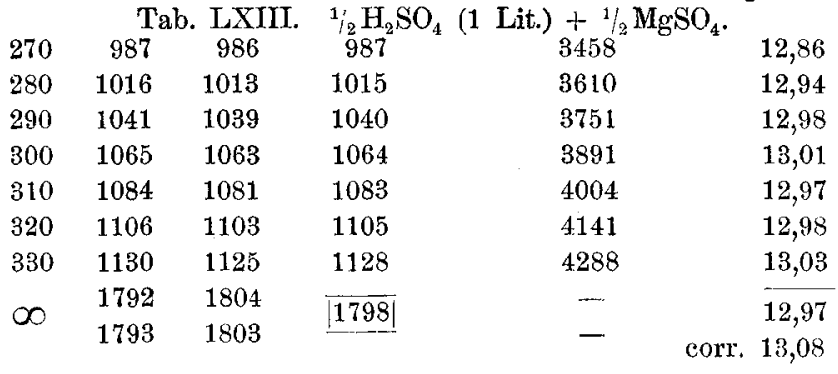


Katalyse d. Methylacetats durch Chlorwasserstoffs. etc. 375 Tab. LXIV. $1 / 2 \mathrm{H}_{2} \mathrm{SO}_{4}(1 \mathrm{Lit})+.1 / 2 \mathrm{MgSO}_{4} .5$ fache Verdünnung.

\begin{tabular}{cccccr}
$t$ & \multicolumn{1}{l}{ I } & II & Mittel & $\log b-\log (b-x)$ & Const. \\
1620 & 1072 & 1073 & 1073 & 3961 & 2,47 \\
1630 & 1079 & 1078 & 1079 & 4029 & 2,47 \\
1640 & 1083 & 1083 & 1083 & 4053 & 2,47 \\
1650 & 1086 & 1085 & 1086 & 4071 & 2,47 \\
1660 & 1088 & 1090 & 1089 & 4090 & 2,47 \\
1670 & 1093 & 1093 & 1093 & 4115 & 2,47 \\
1680 & 1098 & 1100 & 1099 & 4153 & 2,47 \\
$\infty$ & 1786 & 1784 & $\overline{1785}$ & - & 2,47 \\
& 1786 & 1783 & $\underline{ }$ & - & corr. 2,48
\end{tabular}

Die Reactionsgeschwindigkeit der Schwefelsäure wird wie bei der Salzsäure in den untersuchten Fällen durch die Gegenwart verschiedener Salze in verschiedenem Maasse beeinflusst. Hinsichtlich ihrer vermindernden Wirkung auf die Reactionsgeschwindigkeit der Schwefelsäure ergiebt sich aber für obige Salze folgende Reihenfolge, umgekehrt wie bei der Salzsäure, d. h. der vermindernde Einfluss scheint für die Gruppe der Alkalien mit steigendem Atomgewicht zu wachsen.

\begin{tabular}{|c|c|c|c|c|}
\hline $\begin{array}{l}1 / 2 \mathrm{H}_{22} \mathrm{SO}_{4} \\
\text { (1 Lit.) }\end{array}$ & $\begin{array}{l}1 / 2 \mathrm{H}_{2} \mathrm{SO}_{4} \text { (1Lit.) } \\
+1 / 2 \mathrm{MgSO}_{4}\end{array}$ & $\begin{array}{c}1{ }_{2} \mathrm{H}_{2} \mathrm{SO}_{4} \text { (ILit) } \\
+1 / 2 \mathrm{Li}_{2} \mathrm{SO}_{4}\end{array}$ & $\left\{\begin{array}{c}1_{2} \mathrm{H}_{22} \mathrm{SO}_{4} \text { (1Lit.) } \\
+1_{2} \mathrm{Na}_{2} \mathrm{SO}_{4}\end{array}\right.$ & $\mid \begin{array}{c}1 / 2 \mathrm{H}_{2} \mathrm{SO}_{4} \text { (1Lit.) } \\
+1 / 2 \mathrm{~K}_{2} \mathrm{SO}_{4}\end{array}$ \\
\hline 1,000 & 0,787 & 0,620 & 0,579 & 0,530 \\
\hline
\end{tabular}

Dieses verschiedenartige Verhalten der Chlorwasserstoffund Schwefelsäure, der Repräsentanten ein- und zweibasischer Säuren, veranlasste mich, bald nach Beginn dieser Arbeit, entsprechende Versuche mit der Unterschwefelsäure anzustellen. Da nun die Unterschwefelsäure durch ihre Salze in demselben Sinne, wie die Chlorwasserstoffsäure beeinfiusst wird, so glaubte ich, auf Grund dieses Verhaltens die Unterschwefelsäure als einbasische Säure ansprechen zu können. Denn obwohl bereits früher das abweichende Verhalten schwacher einbasischer Säuren von W. Ostwald beobachtet worden, so lag doch kein Grund vor, für die starken einund zweibasischen Säuren eine solche Abweichung vorauszusetzen. Durch eine inzwischen von J. Spohr ${ }^{1}$ ) publicirte

1) Dies. Journ. [2] 32, 32. (1885.) 
376 Trey: Ueb. d. Einfluss einiger Neutralsalze auf die Arbeit, in welcher derselbe auch die Methylendisulfonsäure untersuchte, ergab sich für letztere, dass sie bei Gegenwart ihres Kalisalzes eine Zuckerlösung schneller invertirte, als ohne dasselbe. Ich habe daher die Methylendisulfonsäure, sowie die Dichloressigsäure ebenfalls untersucht und folgende Werthe gefunden.

14. Methylendisulfonsäure.

Tab. LXV. $\quad{ }_{1 / 2} \mathrm{CH}_{2}\left(\mathrm{SO}_{3} \mathrm{H}\right)_{2}$ (2 Lit.)

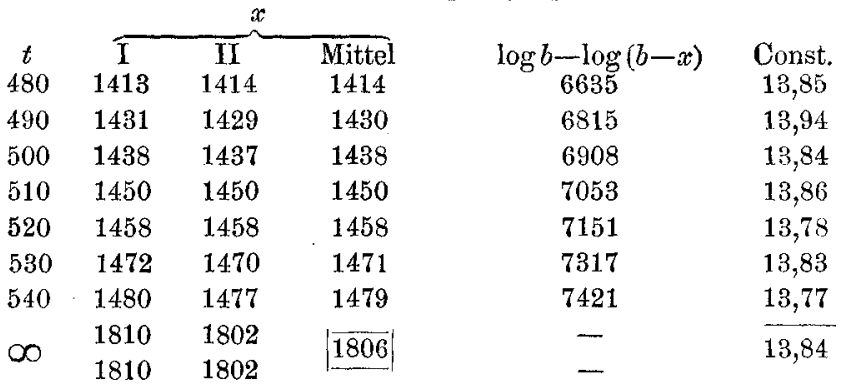

15. Methylendisulfonsäure und methylendisulfonsaures Natrium.

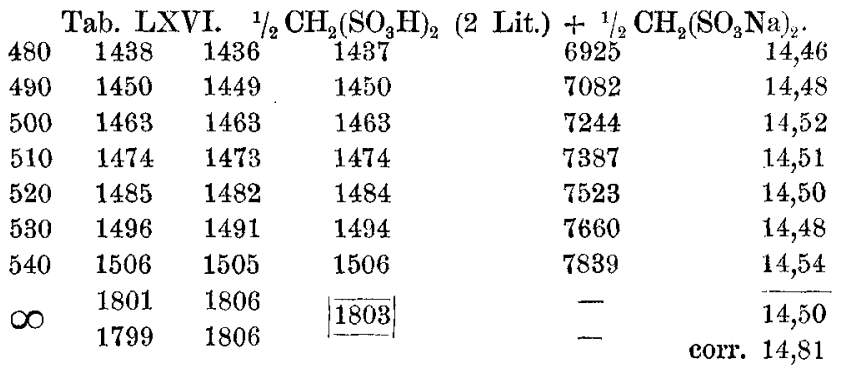

\begin{tabular}{rrrrcr}
\multicolumn{5}{c}{ 16. } & Dichloresssigsäure. \\
960 & 1061 & 1054 & 1058 & 3866 & \\
970 & 1066 & 1062 & 1064 & 3902 & 4,03 \\
980 & 1075 & 1067 & 1071 & 3949 & 4,03 \\
990 & 1081 & 1076 & 1079 & 3991 & 4,03 \\
1000 & 1087 & 1082 & 1085 & 4029 & 4,04 \\
1010 & 1095 & 1089 & 1092 & 4071 & 4,03 \\
1020 & 1101 & 1094 & 1098 & 4109 & 4,04 \\
$\infty$ & 1797 & 1793 & 1795 & - & 4,03 \\
& 1796 & 1791 & $\underline{179}$ & - & 4,03
\end{tabular}


Katalyse d. Methylacetats durch Chlorwasserstoffs. etc. 377

17. Dichloressigsäure und dichloressigsaures Natrium.

Tab. LXVIII. $\mathrm{CHCl}_{2} \mathrm{COOH}\left(2\right.$ Lit.) $+\mathrm{CHCl}_{2} \mathrm{COONa}$.

\begin{tabular}{|c|c|c|c|c|c|}
\hline$t$ & $\mathrm{I}$ & $\overline{\mathrm{II}}$ & $\overline{\text { Mittel }}$ & $\log b--\log (b-x)$ & Const. \\
\hline 960 & 554 & 547 & 551 & 1620 & 1,67 \\
\hline 970 & 559 & 552 & 556 & 1638 & 1,69 \\
\hline 980 & 562 & 558 & 560 & 1652 & 1,69 \\
\hline 1160 & 640 & 637 & 639 & 1945 & 1,68 \\
\hline 1170 & 645 & 641 & 643 & 1961 & 1,68 \\
\hline 1180 & 651 & 643 & 647 & 1976 & 1,68 \\
\hline 1250 & 680 & 673 & 677 & 2094 & 1,68 \\
\hline \multirow{2}{*}{$\infty$} & 1777 & 1765 & \multirow[t]{2}{*}{1770} & - & 1,68 \\
\hline & 1778 & 1760 & & $\mathrm{co}$ & . 1,73 \\
\hline
\end{tabular}

In der That zeigt die zweibasische Methylendisulfonsäure ein der Chlorwasserstoffsäure entsprechendes Verhalten, die Dichloressigsäure dagegen verhält sich wie die Schwefelsäure. - Das der Chlorwasserstoffsäure analoge Verhalten der Unterschwefelsäure bei Gegenwart ihrer Neutralsalze kann also nicht als endgiltiges Kriterium für die Basicität der Unterschwefelsäure gelten, wohl aber scheint daraus hervorzugehen, dass dieselbe in wässriger Liösung keine sauren Salze bildet. Bemerkenswerth bleibt dabei immerhin, dass auch bisher noch keine experimentellen Beweise dafür beigebracht worden, dass die Unterschwefelsäure zwcibasisch sei.

Die Ergebnisse vorliegender Arbeit sind wesentlich folgende :

Die Reactionsgeschwindigkeit der Chlorwasserstoffsäure wird durch die Gegenwart ihrer Salze verstärkt, die der Schwefelsäure durch dieselben vermindert. Numerische Beziehungen für den Einfluss verschiedener Salze ergeben sich nicht; scheint auch eine gewisse Abhängigkeit rom Atomgewicht statt zu haben, so können doch infolge der geringen Anzahl der untersuchten Salze keine allgemeinen Schlüsse gezogen werden. - Mit freundlicher Bereitwilligkeit hat Herr Prof. Dr. W. Ostwald mich auch bei dieser Arbeit unterstützt, wofür ich ihm an dieser Stelle meinen besten Dank ausspreche.

Riga, Polytechnicum, April 1886. 